\title{
川桂皮中具有免疫调节活性的甾体和苯丙素类化学成分
}

\author{
魏夏兰 ${ }^{a}$ 舒朋华 ${ }^{a}$ 刘婷婷 ${ }^{a}$ 向 明 ${ }^{a}$ 张锦文 ${ }^{b}$ \\ 薛永波 ${ }^{a}$ 罗增伟 ${ }^{a}$ 姚广民*, $a$ 张勇慧 $*, a$ \\ ( ${ }^{a}$ 华中科技大学同济医学院药学院 武汉 430030) \\ ( ${ }^{b}$ 华中科技大学同济医学院附属同济医院 武汉 430030)
}

\begin{abstract}
摘要 对川桂 Cinnamomum wilsonii 皮的化学成分进行了研究，从中分离并鉴定出 1 个新的甾体类化合物, $(3 S, 22 R, 24 R)$-stigmast-5-ene-3 $\beta, 22 \alpha$-diol (1), 和 10 个已知化合物, stigmast-5-ene-3 $3,7 \alpha, 22 \alpha$-triol (2), 24-ethylcholest-5-ene$3 \beta, 4 \beta, 22 \alpha$-triol (3), stigmast-5-ene-3 $\beta, 7 \alpha$-diol (4), $\beta$-谷甾醇(5), $\beta$-胡萝卜苷(6), $(1 R, 2 R)$-1-(4-hydroxy-3-methoxyphenyl)1,2,3-propanetriol (7), (1S,2S)-1-(4-hydroxy-3-methoxyphenyl)-1,2,3-propanetriol (8), 丁子香酚(9), 甲氧基丁子香酚(10)和 methyl (E)-ferulate (11). 通过波谱分析和 $\mathrm{Cu}$ 靶单晶 $\mathrm{X}$ 射线衍射方法确定了新化合物 $\mathbf{1}$ 的结构及其绝对构型. 测试了化 合物 $1 \sim 11$ 的体外免疫调节活性, 1,5 和 6 能显著抑制刀豆 $\mathrm{A}$ 诱导的小鼠 $\mathrm{T}$ 细胞增殖, 6 和 9 能显著抑制脂多糖诱导的 小鼠 B 细胞增殖.
\end{abstract}

关键词 川桂; $(3 S, 22 R, 24 R)$-stigmast-5-ene-3 $\beta, 22 \alpha$-diol; 单晶 X 射线衍射; 绝对构型; 免疫调节活性

\section{Steroids and Phenylpropanoids with Immunomodulatory Activities from the Stem Barks of Cinnamomum wilsonii}

\author{
Wei, Xialan $^{a} \quad$ Shu, Penghua $^{a} \quad$ Liu, Tingting $^{a} \quad$ Xiang, Ming $^{a} \quad$ Zhang, Jinwen $^{b}$ \\ Xue, Yongbo $^{a} \quad$ Luo, Zengwei ${ }^{a} \quad$ Yao, Guangmin ${ }^{*, a} \quad$ Zhang, Yonghui ${ }^{*, a}$ \\ ( ${ }^{a}$ School of Pharmacy, Tongji Medical College, Huazhong University of Science and Technology, Wuhan 430030) \\ $\left({ }^{b}\right.$ Tongji Hospital Affiliated to Tongji Medical College, Huazhong University of Science and Technology, Wuhan 430030)
}

\begin{abstract}
A new steroid, $(3 S, 22 R, 24 R)$-stigmast-5-ene-3 $\beta, 22 \alpha$-diol (1), together with ten known compounds, stig-

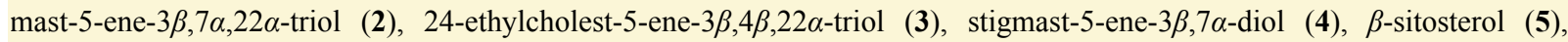
$\beta$-daucosterol (6), (1R,2R)-1-(4-hydroxy-3-methoxyphenyl)-1,2,3-propanetriol (7), (1S,2S)-1-(4-hydroxy-3-methoxyphenyl)1,2,3-propanetriol (8), eugenol (9), methoxyeugenol (10), and methyl $(E)$-ferulate (11) were isolated from the stem barks of Cinnamomum wilsonii. The structure including the absolute configuration of $\mathbf{1}$ was determined by spectroscopic data interpretation and X-ray single-crystal diffraction analysis with $\mathrm{Cu} \mathrm{K} \alpha$ irradiation. Compounds $\mathbf{1} \sim \mathbf{1 1}$ were evaluated for immunomodulatory activities against murine lymphocytes. $\mathbf{1}, \mathbf{5}$ and $\mathbf{6}$ significantly inhibited the proliferation of murine ConA-induced $\mathrm{T}$ cells, and $\mathbf{6}$ and $\mathbf{9}$ significantly inhibited the proliferation of LPS-induced murine B cells.
\end{abstract}

Keywords Cinnamomum wilsonii; $(3 S, 22 R, 24 R)$-stigmast-5-ene-3 $\beta, 22 \alpha$-diol; X-ray single-crystal diffraction; absolute configuration; immunomodulatory activity

川桂(Cinnamomum wilsonii)为樟科樟属植物, 常绿 乔木, 生长在山谷或山坡阳处、沟边, 疏林或密林中, 是 我国特有植物，主产陕西、四川、湖北、湖南、广西、 广东及江西等地 ${ }^{[1]}$. 川桂是一种具有较高经济利用价值 的药食两用植物. 川桂的叶、枝和皮都可用作烹饪香料,
烹制各种肉类、鱼类等食品，具有去腥增香的作用．川 桂皮入药，功效补肾和散寒祛风，治疗风湿筋骨痛、跌 打及腹痛吐江等症 ${ }^{[2]}$. 但是迄今为止，川桂皮的化学成 分还未见文献报道. 为了充分挖掘和利用我国丰富的川 桂资源, 我们首次对川桂皮的化学成分进行了系统研

\footnotetext{
*E-mail: gyap@hust.edu.cn; zhangyh@mails.tjmu.edu.cn

Received March 31, 2013; revised April 28, 2013; published online May 31, 2013.

Project supported by the Scientific Research Foundation for the Returned Oversea Chinese Scholars, Ministry of Education (No. 2010-1561, 40th) and the Program for New Century Excellent Talents in University, Ministry of Education (No. NCET-2008-0224).

教育部留学回国人员科研启动基金(No. 2010-1561，第 40 批)、教育部新世纪优秀人才计划(No. NCET-2008-0224)资助项目.
} 
究, 从中分离并鉴定出 11 个化合物, 其中化合物 $\mathbf{1}$ 为新 化合物, 并测试了所得 11 个化合物的体外免疫调节活 性, 发现化合物 1,5 和 6 具有显著的免疫抑制活性. 本 文将报道化合物 1 11 的提取、分离、结构鉴定及其免 疫调节活性.

\section{1 结果与讨论}

川桂皮用 $95 \%$ 乙醇提取，乙醇提取物减压浓缩后得 浸膏, 用温水混悬后用乙酸乙酯萃取得乙酸乙酯部位. 乙酸乙酯部位经硅胶、反相硅胶和凝胶 Sephadex LH-20 等反复柱层析分离得到 1 个新的甾体类化合物 1,5 个已 知甾体类化合物 $2 \sim 6$ 和 5 个苯丙素类化合物 7 11(图 $1)$.

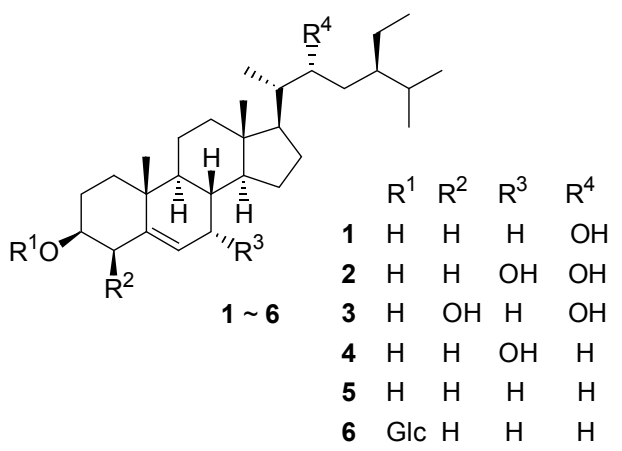

\section{1 新化合物 1 的结构鉴定}

化合物 1 为无色针状结晶, 易溶于氯仿, 微溶于甲 醇. HRESIMS 在 $m / z: 453.3686$ 处给出 $[\mathrm{M}+\mathrm{Na}]^{+}$准分子 离子峰, 推测其分子式为 $\mathrm{C}_{29} \mathrm{H}_{50} \mathrm{O}_{2}$ (calcd. for $\mathrm{C}_{29} \mathrm{H}_{50} \mathrm{O}_{2}$ $\left.\mathrm{Na}^{+}, 453.3703\right)$, 计算得其不饱和度为 5. 化合物 1 的 ${ }^{1} \mathrm{H}$ NMR 谱(表 1)中在 $\delta_{\mathrm{H}} 0.99\left(\mathrm{~s}, \mathrm{H}_{3}-19\right)$ 和 $0.68\left(\mathrm{~s}, \mathrm{H}_{3}-18\right)$ 处 显示 2 个单峰甲基信号, 在 $\delta_{\mathrm{H}} 0.89\left(\mathrm{~d}, J=6.7 \mathrm{~Hz}, \mathrm{H}_{3}-21\right)$, $0.87\left(\mathrm{~d}, J=7.0 \mathrm{~Hz}, \mathrm{H}_{3}-27\right)$ 和 $0.76\left(\mathrm{~d}, J=6.8 \mathrm{~Hz}, \mathrm{H}_{3}-26\right)$ 处 显示 3 个两重峰甲基信号, 在 $0.86\left(\mathrm{~d}, J=7.0 \mathrm{~Hz}, \mathrm{H}_{3}-27\right)$ 处显示 1 个三重峰甲基信号, 在 $\delta_{\mathrm{H}} 3.49$ (dddd, H-3)和 3.68 (br d, H-22)处显示两个连氧次甲基信号，在 $\delta_{\mathrm{H}} 5.32$ $(\mathrm{d}, J=5.0 \mathrm{~Hz}, \mathrm{H}-6)$ 处显示 1 个烯质子信号.

结合 ${ }^{1} \mathrm{H}$ NMR, DEPT 和 HSQC 谱图, ${ }^{13} \mathrm{C} \mathrm{NMR}$ (表 1) 谱图中出现的 29 个碳信号可以归属为 6 个甲基 $\left(\delta_{\mathrm{C}} 12.0\right.$,<smiles>COc1cc(C(O)C(O)CO)ccc1O</smiles><smiles>O=C(Cl)OC1OCC(O)C(O)C(O)C1O</smiles>

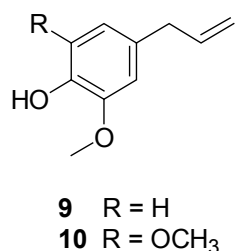<smiles>COC(=O)/C=C/c1ccc(O)c(OC)c1</smiles>

图 1 化合物 1 11 的结构

Figure 1 Structures of compounds 1 11

表 1 化合物 1 的 ${ }^{1} \mathrm{H} \mathrm{NMR}\left(\mathrm{CDCl}_{3}, 400 \mathrm{MHz}\right.$ 和 ${ }^{13} \mathrm{C} \mathrm{NMR}$ 数据 $\left(\mathrm{CDCl}_{3}, 100 \mathrm{MHz}\right)$

Table $1{ }^{1} \mathrm{H} \mathrm{NMR}\left(\mathrm{CDCl}_{3}, 400 \mathrm{MHz}\right)$ and ${ }^{13} \mathrm{C} \mathrm{NMR}\left(\mathrm{CDCl}_{3}, 100 \mathrm{MHz}\right)$ data of compound 1

\begin{tabular}{|c|c|c|c|c|c|}
\hline Position & $\delta_{\mathrm{H}}$, mult, $J$ in $\mathrm{Hz}$ & $\delta_{\mathrm{C}}$ & Position & $\delta_{\mathrm{H}}$, mult, $J$ in $\mathrm{Hz}$ & $\delta_{\mathrm{C}}$ \\
\hline $1 \alpha$ & $1.44 \sim 1.45, \mathrm{~m}$ & 32.1 & $15 \alpha$ & $1.59 \sim 1.60, \mathrm{~m}$ & 24.7 \\
\hline $1 \beta$ & $1.94 \sim 1.96, \mathrm{~m}$ & & $15 \beta$ & $1.08 \sim 1.10, \mathrm{~m}$ & \\
\hline $2 \alpha$ & $1.47 \sim 1.49, \mathrm{~m}$ & 31.9 & $16 \alpha$ & $1.94 \sim 1.96, \mathrm{~m}$ & 40.0 \\
\hline $2 \beta$ & $1.81 \sim 1.82, \mathrm{~m}$ & & $16 \beta$ & $1.15 \sim 1.16, \mathrm{~m}$ & \\
\hline $3 \alpha$ & 3.49 , dddd, $10.7,10.5,4.8,4.4$ & 72.0 & $17 \alpha$ & $1.07 \sim 1.08, \mathrm{~m}$ & 53.2 \\
\hline $4 \alpha$ & $2.27, \mathrm{dd}, 12.9,5.2$ & 42.5 & 18 & $0.68, \mathrm{~s}$ & 12.0 \\
\hline $4 \beta$ & $2.21, \mathrm{dd}, 12.9,10.7$ & & 19 & $0.99, \mathrm{~s}$ & 19.6 \\
\hline 5 & & 141.0 & 20 & $1.24 \sim 1.26, \mathrm{~m}$ & 41.6 \\
\hline 6 & $5.32, \mathrm{~d}, 5.0$ & 121.8 & 21 & $0.89, \mathrm{~d}, 6.7$ & 12.5 \\
\hline $7 \alpha$ & $1.00 \sim 1.02, \mathrm{~m}$ & 30.1 & 22 & 3.68 , br d, 10.0 & 71.5 \\
\hline $7 \beta$ & $1.22 \sim 1.23, \mathrm{~m}$ & & $23 a$ & $1.80 \sim 1.82, \mathrm{~m}$ & 37.5 \\
\hline $8 \beta$ & $1.95 \sim 1.97, \mathrm{~m}$ & 32.1 & $23 b$ & $1.05 \sim 1.07, \mathrm{~m}$ & \\
\hline $9 \alpha$ & $0.90 \sim 0.92, \mathrm{~m}$ & 50.4 & 24 & $1.69 \sim 1.72, \mathrm{~m}$ & 42.7 \\
\hline 10 & & 36.7 & 25 & $1.76 \sim 1.78, \mathrm{~m}$ & 29.0 \\
\hline $11 \alpha$ & $1.48 \sim 1.49, \mathrm{~m}$ & 21.3 & 26 & $0.76, \mathrm{~d}, 6.8$ & 17.8 \\
\hline $11 \beta$ & $1.49 \sim 1.51, \mathrm{~m}$ & & 27 & $0.87, \mathrm{~d}, 7.0$ & 20.8 \\
\hline $12 \alpha$ & $1.34 \sim 1.35, \mathrm{~m}$ & 27.7 & $28 \mathrm{a}$ & $1.33 \sim 1.35, \mathrm{~m}$ & 23.8 \\
\hline $12 \beta$ & $1.69 \sim 1.70, \mathrm{~m}$ & & $28 b$ & $1.23 \sim 1.24, \mathrm{~m}$ & \\
\hline 13 & & 42.9 & 29 & $0.86, \mathrm{t}, 7.0$ & 12.1 \\
\hline $14 \alpha$ & $0.97 \sim 0.99, \mathrm{~m}$ & 56.5 & & & \\
\hline
\end{tabular}


C-18; 12.1, C-29; 12.5, C-21; 17.8, C-26; 19.6, C-19; 20.8, C-27), 10 个亚甲基 $\left(\delta_{\mathrm{C}} 21.3\right.$, C-11; 23.8, C-28; 24.7, C-15; 27.7, C-12; 30.1, C-7; 31.9, C-2; 32.1, C-1; 37.5, C-23; 40.0 , C-16; 42.5, C-4); 7 个次甲基 $\left(\delta_{\mathrm{C}} 29.0, \mathrm{C}-25 ; 32.1\right.$, C-8; 41.6, C-20; 42.7, C-24; 51.4, C-9; 53.2, C-17; 56.5, C-14), 2 个连氧次甲基 $\left(\delta_{\mathrm{C}} 71.5, \mathrm{C}-22 ; 72.0, \mathrm{C}-3\right), 1$ 个 $\mathrm{sp}^{2}$ 杂化次甲基 $\left(\delta_{\mathrm{C}} 121.8, \mathrm{C}-6\right) ; 2$ 个 $\mathrm{sp}^{3}$ 杂化季碳 $\left(\delta_{\mathrm{C}} 36.7\right.$, $\mathrm{C}-10 ; 42.9, \mathrm{C}-13), 1$ 个 $\mathrm{sp}^{2}$ 杂化季碳 $\left(\delta_{\mathrm{C}} 141.0, \mathrm{C}-5\right)$. 双键 占去 1 个不饱和度, 剩余的 4 个不饱和度说明化合物 1 中应该有 4 个环. 因此, 1 是一个四环 $\mathrm{C}_{29}$ 甾体类化合物.

通过分析化合物 $\mathbf{1}$ 的 HSQC 和 ${ }^{1} \mathrm{H}-{ }^{1} \mathrm{H}$ COSY 谱, 可 以推测出化合物含有以下几个结构片段: $\mathbf{a}(\mathrm{C}-1 \sim \mathrm{C}-4)$, b $(\mathrm{C}-6 \sim \mathrm{C}-9-\mathrm{C}-11-\mathrm{C}-12)$, c $(\mathrm{C}-8-\mathrm{C}-14 \sim \mathrm{C}-17-\mathrm{C}-20-$ C-21), d $(\mathrm{C}-20-\mathrm{C}-22 \sim \mathrm{C}-24-\mathrm{C}-28-\mathrm{C}-29)$, e $(\mathrm{C}-24 \sim$ $\mathrm{C}-26)$, 和 $\mathbf{f}(\mathrm{C}-25-\mathrm{C}-27)$ (图 2). 在 HMBC 谱中, $\mathrm{H}-4$ 与双 键 C-5 和 C-6, 烯质子 H-6 与 C-4 的相关峰说明 C-4 接 在双键的 C-5 上. $\mathrm{H}_{3}-19$ 与 C-1, C-5, C-9 和 C-10 的 $\mathrm{HMBC}$ 相关信号说明季碳 C-10 分别与 C-1, C-5, C-9 和 C-19 相连. $\mathrm{H}_{3}-18$ 与 C-12, C-13, C-14, C-17 的 HMBC 相 关信号说明季碳 C-13 分别与 C-12, C-14, C-17 和 C-18 相连. $\mathrm{H}_{3}-21$ 与次甲基 $\mathrm{C}-17$ 和 $\mathrm{C}-20$ 以及连氧次甲基 $\mathrm{C}-22$ 的 HMBC 相关信号说明分子中的另一个羟基应该连在 C-22 上(图 2).

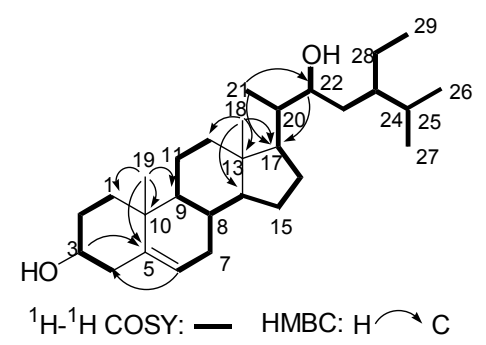

图 2 化合物 $\mathbf{1}$ 的 ${ }^{1} \mathrm{H}-{ }^{1} \mathrm{H}$ COSY 及关键的 HMBC 相关图 Figure $2{ }^{1} \mathrm{H}-{ }^{1} \mathrm{H}$ COSY and key HMBC correlations of compound 1

化合物 1 的环上部分的相对构型可以通过偶合常数 和 NOESY 实验(图 3) 确定. H-3 为 dddd 峰, 它与 H-2 和 $\mathrm{H}-4$ 的偶合常数为 $J=10.7,10.5,4.8,4.4 \mathrm{~Hz}$, 说明 H-3 处在环已烷构象的直立键( $\mathrm{a}$ 键)上, 应该为 $\alpha$ 构型, $3-\mathrm{OH}$ 相应地处在 $\beta$ 方向. $\mathrm{H}_{3}-19$ 与 $\mathrm{H}-2 \beta, \mathrm{H}_{3}-19$ 与 $\mathrm{H}-8 \beta, \mathrm{H}_{3}-19$ 与 $\mathrm{H}-11 \beta, \mathrm{H}_{3}-18$ 与 $\mathrm{H}-8 \beta, \mathrm{H}_{3}-18$ 与 $\mathrm{H}-11 \beta, \mathrm{H}-8 \beta$ 与 $\mathrm{H}-11 \beta$ 的 NOE 相关信号表明 $\mathrm{H}_{3}-19, \mathrm{H}-2 \beta, \mathrm{H}-8 \beta, \mathrm{H}-11 \beta$ 与 $\mathrm{H}_{3}-18$ 的空间取向是一致的. H-20 与 $\mathrm{H}_{3}-18, \mathrm{H}-20$ 与 $\mathrm{H}-16 \beta$ 的 NOE 相关信号表明侧链的空间取向应该为 $\beta$ 位. H- $9 \alpha$ 与 $\mathrm{H}-14 \alpha, \mathrm{H}-14 \alpha$ 与 $\mathrm{H}-17 \alpha$ 的 NOE 相关信号表明 H- $9 \alpha$, $\mathrm{H}-14 \alpha$ 与 $\mathrm{H}-17 \alpha$ 是空间同向的. 仅仅根据 NOESY 分析 还无法确定侧链上 C-20, C-22 和 C-24 的构型.

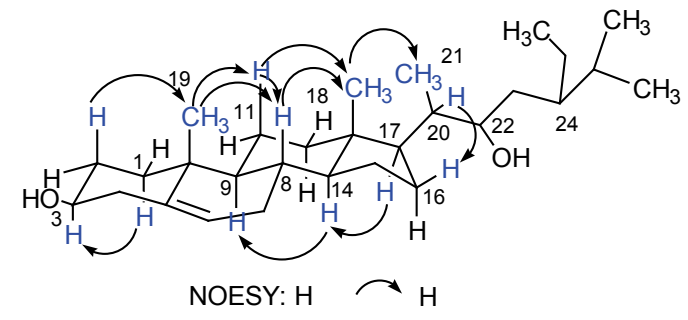

图 3 化合物 $\mathbf{1}$ 的关键的 NOE 相关图

Figure 3 Key NOE correlations of compound 1

文献调研发现，化合物 $\mathbf{1}$ 的平面结构与文献[3]报道 的已知化合物 itesmol 一致. Itesmol 首次由墨西哥学者 Dominguez 等 ${ }^{[4]}$ 于 1963 年从仙人掌科植物 Thelocactus bicolor 中分离得到, 但是文献仅报道了其平面结构. 1973 年, Zanno 等 ${ }^{[3]}$ 采用化学办法, 结合 CD 和 MS, 确 定了 itesmol 的大部分构型, 仍未确定 C-24 的构型. 近 年来, 从楝科捬鸪花属植物 Trichilia quadrijuga 和桑科 拓属植物拓树中也报道了 itesmol, 但是同样没有确定 C-24 的构型 ${ }^{[5,6]}$. 虽然化合物 1 与文献报道的 itesmol 的 理化常数和 NMR 波谱数据都比较接近, 但是甾体侧链 可以自由旋转, 侧链上 C-24 的构型对化合物的旋光度 和 NMR 数据等影响较小, 更重要的是, 1 与 itemsmol 的 来源不同, 所以根据上述数据也不能完全确定化合物 $\mathbf{1}$ 就是已知化合物 itesmol.

为了确定 1 的绝对构型, 特别是甾体侧链上 C-20, C-22 和 C-24 的绝对构型, 我们尝试了在不同条件下培 养单晶. 化合物 1 在甲醇和氯仿混合溶液 $(V: V=3: 1)$ 中室温放置, 最终获得了适合于单晶 $X$ 射线衍射实验晶 体. 挑取 $0.12 \mathrm{~mm} \times 0.10 \mathrm{~mm} \times 0.10 \mathrm{~mm}$ 的晶体在 Bruker SMART APEX-II CCD 衍射仪上采用 $\mathrm{Cu} \mathrm{K} \alpha$ 射线 $(\lambda=$ $1.54178 \AA$ ) 衍射, 得到了 1 的单晶 X 射线衍射结构图(图 4), 验证了其平面结构和相对构型. 经计算, 与绝对构 型相关的 Flack 参数为 0.1 (7), 由此确定 $\mathbf{1}$ 的绝对构型 为 $3 S, 10 R, 13 S, 17 R, 20 S, 22 R, 24 R$.

综上所述, 化合物 $\mathbf{1}$ 的结构确定为 $(3 S, 22 R, 24 R)$ stigmast-5-ene-3 $\beta, 22 \alpha$-diol.

\section{2 已知化合物 2 11 的结构鉴定}

经 ${ }^{1} \mathrm{H} N M R,{ }^{13} \mathrm{C} N M R$, 旋光等波谱学方法分析, 并 与文献对照, 确定已知化合物 $2 \sim 11$ 的结构分别为 stigmast-5-ene-3 $\beta, 7 \alpha, 22 \alpha$-triol $\quad(2)^{[7]}$, 24-ethylcholest-5-ene$3 \beta, 4 \beta, 22 \alpha$-triol (3) ${ }^{[8]}$, stigmast-5-ene- $3 \beta, 7 \alpha$-diol (4) ${ }^{[9]}, \beta$-谷 甾醇 $(5)^{[10]}, \beta$-胡萝卜苷 $(6)^{[11]},(1 R, 2 R)$-1-(4-hydroxy-3methoxyphenyl)-1,2,3-propanetriol $\quad(7)^{[12]}, \quad(1 S, 2 S)-1-(4-$ hydroxy-3-methoxyphenyl)-1,2,3-propanetriol $(\mathbf{8})^{[13]}$, 丁 子香酚 (9) $)^{[14]}$, 甲氧基丁子香酚 $(\mathbf{1 0})^{[14]}$, methyl $(E)$ ferulate (11) ${ }^{[15]}$. 


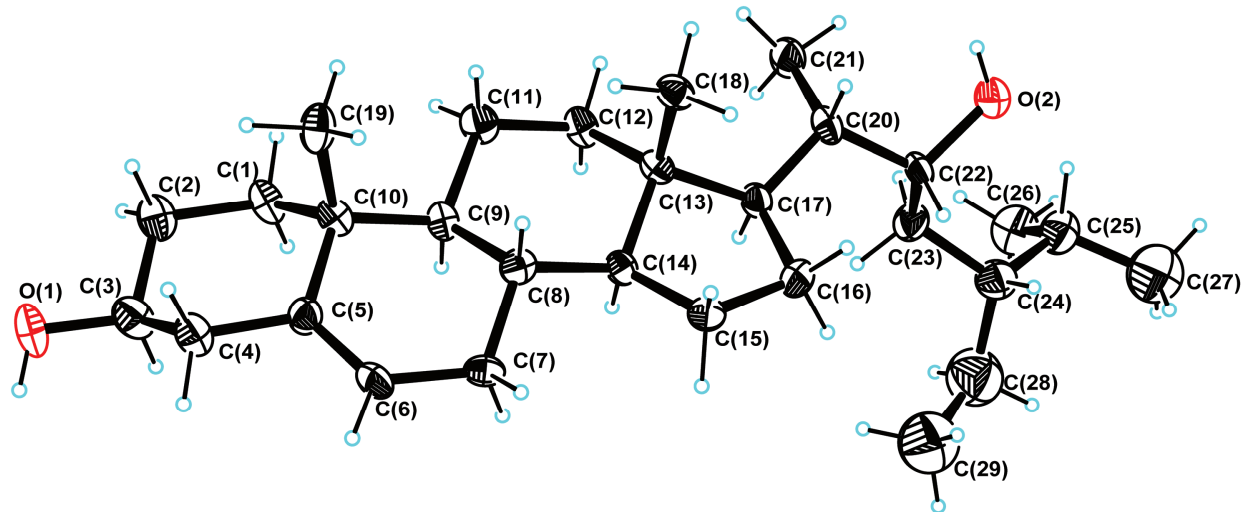

图 4 化合物 1 的单晶 X 射线衍射结构图

Figure 4 X-ray single crystal structure of compound 1

\section{3 化合物 1 11 的免疫调节活性}

文献报道, 已知化合物 $\beta$-谷甾醇(5)及其糖苷 $(6)$ 具 有一定的体内和体外的免疫调节活性 ${ }^{[16]}$. 新化合物 1 是 已知化合物 $\mathbf{5}$ 的 22-差基衍生物, 考虑到结构相似性, 推 测化合物 1 也有免疫调节活性. 我们采用小鼠脾脏淋巴 细胞为模型, 对化合物 1 11 进行了体外免疫调节活性 测试. 结果表明(表 2), 化合物 1 对 ConA 诱导的小鼠 T 细胞增殖具有显著的抑制活性, 抑制率接近阳性药物环 孢素 A (Cyclosporin A, CsA), 且呈现剂量依赖性. 化合 物 5 和 6 对 ConA 诱导的小鼠 $T$ 细胞增殖具有显著的抑 制活性，且在低浓度 $(0.16,0.032$ 和 $0.0064 \mu \mathrm{mol} / \mathrm{L})$ 下抑 制率高于 CsA. 化合物 6 对 LPS 诱导的小鼠 B 细胞增殖 具有显著的抑制活性, 抑制率接近阳性药物 CsA, 且在 中低浓度下抑制率高于 CsA. 化合物 9 在高浓度 40 和 8 $\mu \mathrm{mol} / \mathrm{L}$ )下对 LPS 诱导的小鼠 B 细胞增殖具有一定的抑 制作用. 其它化合物在测试浓度下对 ConA 诱导的小鼠 $\mathrm{T}$ 和 LPS 诱导的小鼠 B 细胞的增殖未表现出显著的抑制 或增强活性.

为了确定活性化合物对 ConA 诱导的小鼠 $\mathrm{T}$ 和 LPS 诱导的小鼠 $\mathrm{B}$ 细胞的增殖抑制是否与细胞毒性有关, 我 们同时测试了同等浓度下化合物对淋巴细胞的细胞毒 性, 发现化合物 1 11 均无明显细胞毒性(表 2). 所以, 化 合物 $1,5,6$ 和 9 表现出来的对 $\mathrm{T}$ 或 $\mathrm{B}$ 细胞的增殖抑制活 性与化合物本身的细胞毒性无关, 免疫抑制活性和作用 机制值得深入研究.

\section{2 结论}

本文利用各种柱层析方法对川桂皮的化学成分进 行了系统研究, 分离出 11 个化合物. 利用波谱方法鉴定 了 11 个化合物的结构, 分别为 1 个新的甾体类化合物, 5 个已知甾体类化合物, 5 个已知的苯丙素类化合物. 以 环孢素 $\mathrm{A}$ (Cyclosporin $\mathrm{A}, \mathrm{CsA}$ )为阳性药物, 评价了 11
表 2 化合物 1 11 对小鼠淋巴细胞增殖的影响

Table 2 Effects of compounds $\mathbf{1} \sim \mathbf{1 1}$ on murine lymphocyte proliferation

\begin{tabular}{|c|c|c|c|c|}
\hline \multirow{2}{*}{ 化合物 } & \multirow{2}{*}{$\begin{array}{c}\text { 浓度/ } \\
\left(\mu \mathrm{mol} \cdot \mathrm{L}^{-1}\right)\end{array}$} & \multirow[b]{2}{*}{$\begin{array}{l}\text { 淋巴细胞- } \\
\text { 存活率/\% }\end{array}$} & \multicolumn{2}{|c|}{ 增殖抑制/增强率 ${ }^{a} / \%$} \\
\hline & & & $\begin{array}{c}\text { ConA 诱导的 } \\
\mathrm{T} \text { 细胞 }\end{array}$ & $\begin{array}{c}\text { LPS 诱导的 } \\
\text { B 细胞 }\end{array}$ \\
\hline \multirow{5}{*}{1} & 40 & 109 & -44 & -19 \\
\hline & 8 & 104 & -42 & -10 \\
\hline & 0.16 & 108 & -13 & -9 \\
\hline & 0.032 & 107 & -9 & -3 \\
\hline & 0.0064 & 107 & -8 & -1 \\
\hline \multirow{5}{*}{2} & 40 & 100 & -5 & -4 \\
\hline & 8 & 101 & -5 & -4 \\
\hline & 0.16 & 102 & -4 & -2 \\
\hline & 0.032 & 101 & -4 & -1 \\
\hline & 0.0064 & 101 & -1 & +1 \\
\hline \multirow{5}{*}{3} & 40 & 100 & -6 & -3 \\
\hline & 8 & 100 & -5 & -2 \\
\hline & 0.16 & 100 & -4 & -2 \\
\hline & 0.032 & 100 & -4 & -1 \\
\hline & 0.0064 & 101 & -1 & 0 \\
\hline \multirow{5}{*}{4} & 40 & 96 & -2 & -11 \\
\hline & 8 & 100 & +2 & -3 \\
\hline & 0.16 & 96 & +2 & -4 \\
\hline & 0.032 & 95 & +2 & -3 \\
\hline & 0.0064 & 98 & +1 & -2 \\
\hline \multirow{5}{*}{5} & 40 & 100 & -29 & -4 \\
\hline & 8 & 100 & -27 & -6 \\
\hline & 0.16 & 100 & -27 & -5 \\
\hline & 0.032 & 99 & -26 & -4 \\
\hline & 0.0064 & 99 & -15 & -3 \\
\hline \multirow{5}{*}{6} & 40 & 100 & -35 & -35 \\
\hline & 8 & 101 & -30 & -32 \\
\hline & 0.16 & 99 & -26 & -32 \\
\hline & 0.032 & 101 & -21 & -30 \\
\hline & 0.0064 & 102 & -12 & -26 \\
\hline
\end{tabular}




\begin{tabular}{|c|c|c|c|c|}
\hline \multirow[b]{2}{*}{ 化合物 } & \multirow{2}{*}{$\begin{array}{c}\text { 浓度/ } \\
\left(\mu \mathrm{mol} \cdot \mathrm{L}^{-1}\right)\end{array}$} & \multirow{2}{*}{$\begin{array}{l}\text { 淋巴细胞- } \\
\text { 存活率 } / \%\end{array}$} & \multicolumn{2}{|c|}{ 增殖抑制/增强率 ${ }^{a} / \%$} \\
\hline & & & $\begin{array}{c}\text { ConA 诱导的 } \\
\mathrm{T} \text { 细胞 }\end{array}$ & $\begin{array}{c}\text { LPS 诱导的 } \\
\text { B 细胞 } \\
\end{array}$ \\
\hline \multirow{5}{*}{7} & 40 & 100 & -10 & -9 \\
\hline & 8 & 100 & -9 & -6 \\
\hline & 0.16 & 98 & -8 & -5 \\
\hline & 0.032 & 100 & -6 & -2 \\
\hline & 0.0064 & 100 & -4 & +1 \\
\hline \multirow{5}{*}{8} & 40 & 99 & -5 & -9 \\
\hline & 8 & 100 & -2 & -7 \\
\hline & 0.16 & 100 & -4 & -8 \\
\hline & 0.032 & 100 & -4 & -7 \\
\hline & 0.0064 & 100 & -5 & -6 \\
\hline \multirow{5}{*}{9} & 40 & 103 & -12 & -24 \\
\hline & 8 & 102 & -10 & -20 \\
\hline & 0.16 & 102 & -8 & -11 \\
\hline & 0.032 & 100 & -5 & -9 \\
\hline & 0.0064 & 100 & -4 & -1 \\
\hline \multirow{5}{*}{10} & 40 & 100 & -2 & -1 \\
\hline & 8 & 100 & -1 & 0 \\
\hline & 0.16 & 100 & -2 & 0 \\
\hline & 0.032 & 101 & -2 & 0 \\
\hline & 0.0064 & 100 & -1 & 0 \\
\hline \multirow{5}{*}{11} & 40 & 101 & -15 & -8 \\
\hline & 8 & 100 & -13 & -7 \\
\hline & 0.16 & 101 & -12 & -5 \\
\hline & 0.032 & 99 & -7 & -2 \\
\hline & 0.0064 & 100 & -5 & +1 \\
\hline \multirow{5}{*}{ CsA } & 40 & 101 & -51 & -37 \\
\hline & 8 & 101 & -31 & -28 \\
\hline & 0.16 & 102 & -21 & -19 \\
\hline & 0.032 & 100 & -14 & -14 \\
\hline & 0.0064 & 102 & -6 & -7 \\
\hline
\end{tabular}

$\bar{a}-:$ 代表抑制作用, + : 代表增强作用.

个化合物的体外免疫调节活性, 化合物 1,5 和 6 能显著 抑制刀豆 $\mathrm{A}(\mathrm{ConA})$ 诱导的 $\mathrm{T}$ 细胞增殖, 化合物 6 能显著 抑制脂多糖(LPS)诱导的 B 细胞增殖, 化合物 9 对脂多糖 (LPS)诱导的 B 细胞增殖有一定的抑制作用, 为进一步 开发利用川桂皮提供了科学依据.

\section{3 实验部分}

\section{1 仪器与试剂}

旋光度由 Perkin-Elmer 341 型数字旋光仪测定. 熔 点由 Beijing Tech X-5 型熔点仪测定. NMR 由 Bruker AM-400 核磁共振仪测定; ${ }^{1} \mathrm{H}$ NMR 位移值以気代溶剂中 残存的 $\mathrm{CHCl}_{3}\left(\delta\right.$ 7.24) 为内标, ${ }^{13} \mathrm{C} \mathrm{NMR}$ 位移值以 $\mathrm{CDCl}_{3}$ $(\delta$ 77.23)为内标. HRESIMS 由 Thermo Fisher LC-LTQOrbitrap XL spectrometer 质谱仪测定. X 射线晶体衍射
在 Bruker SMART APEX-II CCD 衍射仪上进行, 采用经 石墨单色器单色化的 $\mathrm{CuK} \alpha$ 射线 $(\lambda=1.54178 \AA)$. Sephadex LH-20 由 GE Healthcare Bio-Sciences AB 生产. 层析用硅胶(200 300 目)及 TLC 预制板(G60 F-254)由 烟台江友硅胶开发有限公司提供. 各种溶剂均为分析 纯，由国药集团化学试剂有限公司提供.

\section{2 植物材料}

川桂 C. wilsonii 皮 2010 年 7 月采自四川省巫溪，由 华中科技大学同济医学院药学院张长弓教授鉴定. 样品 标本(No. 2010-0702)保存在中国湖北省华中科技大学同 济医学院药学院天然药物化学教研室.

\section{3 提取与分离}

干燥川桂皮 $15 \mathrm{~kg}$, 粉碎, 用 $95 \%$ 乙醇冷浸提取 3 次, 减压回收乙醇, 得到总浸亳 $800 \mathrm{~g}$. 将总浸亳加适量 蒸馏水混悬, 用乙酸乙酯萃取, 减压浓缩, 得到浸膏 $348 \mathrm{~g}$. 将此浸亳经硅胶柱, 以石油醚一丙酮 $(V: V=50$ : $1 \sim 0: 1)$ 进行洗脱, 得到 5 个部分 $(F r .1 \sim$ Fr. 5$)$. 经反复 硅胶、反相硅胶、凝胶 Sephadex LH-20 柱层析进一步纯 化, 从 Fr.1 中分离得到化合物 $\mathbf{1}(30 \mathrm{mg})$. 从 Fr.2 得到化 合物 $\mathbf{4}(22 \mathrm{mg}), \mathbf{5}(208 \mathrm{mg})$ 和 $\mathbf{1 1}(5 \mathrm{mg})$. 从 Fr.3 得到化合 物 $9(12 \mathrm{mg})$ 和 $\mathbf{1 0}(20 \mathrm{mg})$, 从 Fr.4 得到化合物 $\mathbf{2}(20 \mathrm{mg})$, $3(11 \mathrm{mg}$ )和 $7(15 \mathrm{mg})$. Fr.5 经过凝胶柱层析(Sephadex LH-20, 甲醇)得到化合物 $6(87 \mathrm{mg})$ 和 $8(12 \mathrm{mg})$.

\section{$3.4(3 S, 22 R, 24 R)$-stigmast-5-ene-3 $\beta, 22 \alpha$-diol (1)的} 理化常数及波谱数据

无色针状结晶，易溶于氯仿，微溶于甲醇. m.p. $175 \sim 176{ }^{\circ} \mathrm{C} .[\alpha]_{\mathrm{D}}^{25}-21.6\left(\right.$ c $\left.1.3, \mathrm{CHCl}_{3}\right) ;{ }^{1} \mathrm{H}$ NMR $\left(\mathrm{CDCl}_{3}, 400 \mathrm{MHz}\right)$ 和 ${ }^{13} \mathrm{C} \mathrm{NMR}\left(\mathrm{CDCl}_{3}, 100 \mathrm{MHz}\right)$ 数据见 表 1. HRESIMS calcd for $\mathrm{C}_{29} \mathrm{H}_{50} \mathrm{O}_{2} \mathrm{Na}[\mathrm{M}+\mathrm{Na}]^{+}$ 453.3703, found 453.3686 .

\section{$3.5(3 S, 22 R, 24 R)$-stigmast-5-ene-3 $\beta, 22 \alpha$-diol (1) 的 晶体结构测定}

将化合物在甲醇和氯仿 $(V: V=3: 1)$ 混合溶液中 室温放置得结晶, 得无色晶体, 该晶体的分子式为 $\mathrm{C}_{29} \mathrm{H}_{50} \mathrm{O}_{2} \cdot \mathrm{CH}_{3} \mathrm{OH}\left(M_{\mathrm{r}}=462.73\right)$; m.p. $175 \sim 176{ }^{\circ} \mathrm{C}$; 属于 斜方晶系, 空间群 $P 22_{1} 2_{1} 2_{1}$; 晶胞参数: $a=6.0951(4) \AA$, $b=15.0947(8) \AA, c=33.5288$ (19) $\AA, \alpha=\gamma=\beta=90^{\circ}, V=$ $3084.8(3) \AA^{3}, Z=4, F(000)=1032, D_{\mathrm{c}}=0.996 \mathrm{Mg} / \mathrm{m}^{3}$.

晶体 $(0.12 \mathrm{~mm} \times 0.10 \mathrm{~mm} \times 0.10 \mathrm{~mm})$ 结构的测定在 Bruker SMART APEX-II CCD 衍射仪上进行. 采用经石 墨单色器单色化的 $\mathrm{Cu} \mathrm{K} \alpha$ 射线 $(\lambda=1.54178 \AA)$. 于 $296(2)$ $\mathrm{K}$, 在 $3.94^{\circ}<\theta<40.59^{\circ}$ 范围内收集 9890 个衍射点; 其 中, 独立衍射点 1881 个 $\left(R_{\mathrm{int}}=0.0366\right)$. 晶体结构用直接 法(SIR97 $\left.{ }^{[17]}\right)$ 解出, 并用全矩阵最少二乘法 $\left(\mathrm{SHELXL}^{[18]}\right)$ 
对所有非氢原子各向异性热参数进行修正. 所有非氢原 子都给出了各向异性热参数. 氢原子的位置由理论加氢 得出. $R_{1}=0.0798, w R_{2}=0.2321[I>2 \sigma(I)]$, Flack 参数为 0.1 (7). 晶体数据保存在英国剑桥数据中心, 保存号 CCDC 931377.

\section{6 免疫调节活性测试}

\subsection{1 实验动物}

$\mathrm{BALB} / \mathrm{c}$ 纯系小鼠, 雄性, $18 \sim 20 \mathrm{~g}$, 购自湖北省实 验动物研究中心, 动物生产许可证号: $\operatorname{SCXK}($ 鄂) 2008-0005 号. 所有实验均严格按照实验动物有关条例 进行.

\subsection{2 小鼠脾脏淋巴细胞的制备}

参照文献[19]中的方法制备.

3.6.3 CCK-8 法检测化合物对小鼠脾脏淋巴细胞活 性的影响

小鼠脾脏淋巴细胞悬液 $1 \times 10^{6}$ 孔接种于 96 孔板, 同时加入不同浓度化合物, 另设相应的溶媒对照及培养 液本底对照, 总体积为 $200 \mu \mathrm{L} .37^{\circ} \mathrm{C}, 5 \% \mathrm{CO}_{2}$ 培养箱中 培养 48 h. 结束培养前 $8 \sim 10 \mathrm{~h}$ 加入 CCK-8 溶液. 至培 养结束, 于酶标仪 $450 \mathrm{~nm}$ (参比 $650 \mathrm{~nm}$ ) 处测定 OD 值.

3.6.4 CCK-8 法检测化合物对小鼠脾脏 T, B 淋巴细 胞增殖功能的影响

小鼠脾脏淋巴细胞悬液 $5 \times 10^{5}$ 孔接种于 96 孔板, 加入 ConA(终浓度 $5 \mu \mathrm{g} / \mathrm{mL}$ )或 LPS(终浓度 $10 \mu \mathrm{g} / \mathrm{mL}$ ), 不同浓度化合物, 并设相应的无 ConA 或 LPS 对照孔作 为阴性对照组, 仅加入 ConA 或 LPS 而无化合物对照孔 作为阳性对照组. $37{ }^{\circ} \mathrm{C}, 5 \% \mathrm{CO}_{2}$ 培养箱中培养 48 h. 结 束培养前 $8 \sim 10 \mathrm{~h}$ 加入 CCK-8 溶液. 至培养结束, 于酶 标仪 $450 \mathrm{~nm}$ (参比 $650 \mathrm{~nm}$ ) 处测定 OD 值.

\subsection{5 结果评定}

细胞存活率 $(\%)=\left[\right.$ 化合物组 $\left(\mathrm{OD}_{450}\right) /$ 阴性对照组 $\left.\left(\mathrm{OD}_{450}\right)\right] \times 100$. 增强/抑制率 $(\%)=\left[\left(\right.\right.$ 化合物组 $\left(\mathrm{OD}_{450}\right)-$ 阳性对照组 $\left.\left(\mathrm{OD}_{450}\right)\right) /\left(\right.$ 阳性对照组 $\left.\left(\mathrm{OD}_{450}\right)\right] \times 100$. 在无细 胞毒性作用的情况下，增强/抑制率达到 $10 \%$ 或以上，则 说明该样品有增强/抑制小鼠淋巴细胞的作用.

致谢 感谢华中科技大学同济医学院药学院张长弓教 授鉴定植物材料, 华中师范大学孟祥高老师测试化合物 1 的单晶 $X$ 射线衍射.

辅助材料 (Supporting Information) 化合物 1 的 HRESIMS, ${ }^{1} \mathrm{H}$ NMR, ${ }^{13} \mathrm{C}$ NMR, DEPT, HSQC, HMBC, ${ }^{1} \mathrm{H}-{ }^{1} \mathrm{H}$ COSY 和 NOESY 谱图; 晶体堆积图; 晶体学及结
构修正数据; 选择性键长和键角数据; 氢键数据; 扭转 角数据. 这些材料可以免费从本刊网站 (http://siocjournal.cn/)上下载.

\section{References}

[1] Xiao, P. G. Modern Chinese Materia Medica, Vol. 3, Chemical Industry Press, Beijing, 2002, p. 645 (in Chinese). (肖培根, 新编中药志, 第 3 卷, 化工出版社, 北京, 2002, p. 645.)

[2] Li, X. W. Flora of China, Vol. 31, Science Press, Beijing, 1982, p. 213 (in Chinese) (李锡文, 中国植物志, 第 31 卷, 科学出版社, 北京, 1982, p. 213.)

[3] Zanno, P. R.; Nakanishi, K.; Morales, J. G.; Dominguez, X. A. Steroids 1973, 22, 829 .

[4] Dominguez, X. A.; Barragan, V. A.; Leon V. J. O.; Krause, S. L.; Bravo, G. A.; Morales, G. J. Planta Med. 1968, 16, 458.

[5] Rodrigues, V. F.; Carmo, H. M.; Braz Filho, R.; Mathias, L.; Vieira, I. J. C. Nat. Prod. Commun. 2010, 5, 179.

[6] Wang, A. P.; Liu, M. C.; Yang, S. J.; Hu, D. Y; Yang, S. Zhongguo Shiyan Fangjixue Zazhi 2011, 17, 113 (in Chinese). (王安平, 刘明川, 杨胜杰, 胡德禹, 杨松, 中国实验方剂学杂 志, 2011, 17, 113.)

[7] Li, Y.; Ishibashi, M.; Satake, M.; Chen, X.; Oshima, Y.; Ohizumi, Y. J. Nat. Prod. 2003, 66, 696.

[8] Xu, Y. J.; Imiyabir, Z.; Lai, Y. H.; Vittal, J. J.; Goh, S. H. ACGC Chem. Res. Commun. 2001, 13, 37.

[9] Wu, H. B.; Lan, X. C.; Wang, W. S. Nat. Prod. Res. Dev. 2012, 24, 55 (in Chinese). (武海波, 蓝晓聪, 王文蜀, 天然产物研究与开发, 2012, 24, 55.)

[10] Zhao, X. M.; Ye, X. Q.; Xi, Y. F.; Zhu, D. Y.; Jiang, S. H. China J. Chin. Mater. Med. 2003, 28, 237 (in Chinese).

(赵雪梅, 叶兴乾，席屿芳，朱大元，蒋山好，中国中药杂志， 2003, 28, 237.)

[11] Yuan, J. R.; Li, Q. W.; Li, Z. L. China J. Chin. Mater. Med. 2000, 25, 421 (in Chinese).

(袁久荣, 李全文, 李智立，中国中药杂志, 2000, 25, 421.)

[12] Greca, M. D.; Ferrara, M.; Fiorentino, A.; Monaco, P.; Previtera, L. Phytochemistry 1998, 49, 1299

[13] Warashina, T.; Nagatani, Y.; Noro, T. Phytochemistry 2005, 66 , 589.

[14] Song, W. Y.; Ma, Y. B.; Chen, J. J.; Zhou, J.; Jiang, Z. Y.; Chang, X. L.; Zhang, X. M. Chin. Trad. Herb. Drugs 2009, 40, 1216 (in Chinese).

(宋文雍，马云保，陈纪军，周俊，江志勇，常新亮，张雪梅，中 草药, 2009, 40, 1216.)

[15] Miyazawa, M.; Okuno, Y.; Nakamura, S.; Kameoka, H. J. Agric. Food Chem. 1998, 46, 904.

[16] Fraile, L.; Crisci, E.; Cordoba, L.; Navarro, M. A.; Osada, J.; Montoya, M. Int. Immunopharmacol. 2012, 13, 316.

[17] Altomare, A.; Burla, M. C.; Camalli, M.; Cascarano, G. L.; Giacovazzo, C.; Guagliardi, A.; Moliterni, A. G. G.; Polidori, G.; Spagna, R. J. Appl. Crystallogr. 1999, 32, 115.

[18] Sheldrick, G. M. SHELXL97, Program for X-Ray Crystal Structure Refinement, University of Göttingen, Germany, 1997.

[19] Kawaguchi, T.; Matsumoto, I.; Osawa, T. J. Biol. Chem. 1974, 249, 2786.

(Li, L.; Lu, Z.) 